\title{
Impacto en el desenlace funcional en pacientes con ictus: experiencia de una unidad de cuidados neurovasculares
}

\author{
Fernando Góngora-Rivera, Alan Baltazar Treviño-Herrera, Alejandro González-Aquines, Adolfo César \\ Cordero-Pérez, Carlos Rodrigo Cámara-Lemarroy, Adrián Infante-Valenzuela, Erick García-Valadez y \\ Héctor Jorge Villarreal-Velázquez; NeuroVasc-HU* y GECEN Investigators ${ }^{\dagger}$ \\ Departamento de Neurología, Hospital Universitario Dr. José Eleuterio González, Facultad de Medicina, Universidad Autónoma de Nuevo León, \\ Monterrey, Nuevo León, México \\ *NeuroVasc-HU: Alejandro Marfil-Rivera, Héctor Ramón-Martínez, Rómulo Ramírez, Ricardo Rangel-Guerra, Héctor J. Villarreal-Montemayor, \\ Alfonso Gil-Valadez, Xochitl Ortiz, Óscar Cavazos, Victoria Montes, Donato Cantú, Antonio Anaya, Hazael Flores, Héctor Julián-Calderón, Anally \\ Soto-García, Mildred Gutiérrez, Ana Lucía-Herrera, Walter Muruet, Sergio Saldivar-Davila, Daniela Treviño-Herrera y Jonathan Rocha. \\ † GECEN Investigators: Humberto Leal-Bailey, Mezli Espinosa-Ortega, Nicolás Escobedo-Zuñiga, Denisse Martínez-Roque y Mario Cristóbal-Niño.
}

\section{Resumen}

Antecedentes y objetivo: Las unidades de cuidados neurovasculares (UCN) impactan favorablemente en el pronóstico funcional del paciente con ictus en comparación con las salas de internamiento general. La efectividad de las UCN en México no ha sido evaluada. Nuestro objetivo fue determinar el impacto que tiene una UCN en un hospital académico de tercer nivel del noreste de México. Método: Estudio de cohorte prospectivo, observacional y analítico. La población fue dividida en dos periodos: el primero consistió en aquellos pacientes ingresados antes de la implementación de la UCN (2008-2010), y el segundo consistió en pacientes ingresados posterior a la implementación de la UCN (2010-2014). Se evaluó el estado funcional al egreso y a los 3 meses. Resultados y conclusiones: Se incluyeron 598 pacientes (periodo 1: 246; periodo 2: 352). En el periodo 2 se incrementó la profilaxis de trombosis venosa profunda (razón de momios [RM]: 3.235; intervalo de confianza del $95 \%$ [IC $95 \%$ ]: 2.18-4.80; $p=0.01$ ) y se redujeron la estancia hospitalaria (RM: 0.42; IC $95 \%$ : 0.29-0.62; $p=0.01$ ) y la discapacidad funcional grave (Rankin $\geq 3$ ) a los 3 meses de seguimiento (RM: 0.42; IC $95 \%$ : 0.29-0.62; $p=0.01$ ). La implementación de una UCN mejoró el desenlace funcional a 3 meses y disminuyó los días de estancia intrahospitalaria de pacientes con ictus.

\section{PALABRAS CLAVE: Ictus. Unidad neurovascular. Pronóstico funcional.}

\section{Abstract}

Background and objective: Neurovascular care units (NCU) have a positive impact on the functional prognosis of stroke patients. The effectiveness of NCUs in Mexico has not been evaluated. Our objective was to determine the impact of an NCU in a third-level academic hospital in northeastern of Mexico. Method: We performed a prospective observational, analytic cohort study. The population was divided into two periods: the first one consisted of those patients admitted before the implementation of the NCU (2008-2010), and the second period consisted of patients admitted after the implementation of the UCN (2010-2014). Functional status was assessed with the modified Rankin scale at discharge and 3 months. Results and conclusions: 598 patients were included (period 1: 246; period 2: 352). Patients in period 2 had a higher deep venous thrombosis prophylaxis (odds ratio [OR]: 3.235; $95 \%$ confidence interval [95 \% Cl]: 2.18-4.80; $p=0.01$ ), a shorter hospital stay (OR: 0.42; $95 \% \mathrm{Cl}: 0.29-0.62 ; p=0.01$ ) and less severe disability (Rankin $\geq 3$ ) at 3 months of follow-up (OR: 0.42; $95 \% \mathrm{Cl}: 0.29-0.62 ; p=0.01$ ). The implementation of an NCU in a third-level academic hospital improved the functional outcome at 3 months and decreased the days of in-hospital stay of patients with stroke.

\section{KEY WORDS: Stroke. Stroke care unit. Functional outcome.}

Correspondencia:

Fernando Góngora-Rivera

E-mail: fernando.gongora@hotmail.com
Fecha de recepción: 16-08-2018

Fecha de aceptación: 20-08-2018 DOI: 10.24875/GMM.18004579
Gaceta Med Mex. 2018;154(Suppl 2): S56-S60

Disponible en PubMed www.gacetamedicademexico.com 


\section{Introducción}

La enfermedad vascular cerebral es la primera causa de discapacidad y la segunda causa de muerte en el mundo. ${ }^{1}$ Su forma aguda y de presentación súbita es denominada ictus según el diccionario de la Real Academia Española, equivalente al término en inglés stroke. En México, la incidencia acumulada es de 232.2 por cada 100000 personas, mientras que la prevalencia en mayores de 60 años es de 18.2 por cada 1000 personas. ${ }^{2}$ El ictus isquémico, denominado también infarto cerebral, es considerado un problema de salud pública debido a su impacto clínico y socioeconómico. ${ }^{3}$

Las unidades de cuidados neurovasculares (UCN) están especialmente diseñadas para el cuidado integral de pacientes con ictus, y se han asociado con mejores desenlaces clínicos en comparación con las salas de internamiento general (SIG). Diversos metaanálisis han comparado las UCN contra las SIG, demostrando una reducción en la discapacidad y la mortalidad intrahospitalaria en las UCN. ${ }^{4,5}$ Además, la admisión a una UCN incrementa los beneficios asociados a la terapia trombolítica con activador tisular del plasminógeno (aPTr). ${ }^{6}$ Es por ello que las guías clínicas actuales sobre ictus recomiendan el ingreso de los pacientes a las UCN. ${ }^{7}$

El objetivo primario de este estudio fue comparar los desenlaces clínicos en pacientes ingresados antes y después del establecimiento de una UCN. Nuestra hipótesis fue que habría un mejor desenlace funcional al momento del egreso y a los 90 días posterior al egreso hospitalario, así como una reducción en la mortalidad intrahospitalaria y en los días de estancia en el periodo después de la implementación de la UCN.

\section{Método}

\section{Diseño del estudio}

Estudio observacional y analítico, elaborado a partir de una cohorte prospectiva de pacientes con enfermedad vascular cerebral (i-Registro Neurovascular). ${ }^{8}$ Se dividió en dos periodos: el primero previo a la implementación de la UCN (2008-2010) y el segundo con la UCN (2010-2014). Se recibió la aprobación por el Comité Local de Ética (NR-1300) y los pacientes firmaron consentimiento para que su información no identificada fuera incluida en el registro.

\section{Unidad de cuidados neurovasculares}

La UNC se encuentra en el Departamento de Neurología del Hospital Universitario Dr. José E. González, y fue inaugurada el 29 de octubre del año 2010 (Día Mundial del Infarto Cerebral). Consiste en cuatro camas separadas de las SIG, cada una equipada con aparatos de monitoreo electrocardiográfico continuo y bajo el cuidado constante de un equipo de cuidados neurovasculares conformado por enfermería, neurólogos con entrenamiento en enfermedades neurovasculares y al menos cuatro neurólogos o internistas en entrenamiento. Además, cuenta con un equipo de ultrasonido Doppler carotídeo, ultrasonido Doppler transcraneal y Holter de ritmo cardiaco.

\section{Desenlaces}

Se consideró la escala de Rankin modificada (mRs) para evaluar la funcionalidad al egreso y a los 3 meses, y la mortalidad intrahospitalaria (Tabla 2). Los puntajes de la mRs fueron dicotomizados en desenlace bueno $(m R s \leq 2)$ y pobre $(m R s \geq 3) .{ }^{9}$

Se incluyeron variables sociodemográficas, factores de riesgo cardiovasculares e índice de comorbilidad de Charlson. Otras variables analizadas y usadas como desenlaces secundarios fueron el tiempo desde el inicio de los síntomas hasta la puerta del hospital, la escala de ictus NIHSS (National Institute of Health Stroke Scale) al ingreso y al egreso, la etiología del ictus, la medicación, las complicaciones intrahospitalarias y los días de estancia hospitalaria.

\section{Análisis estadístico}

Se realizó un análisis descriptivo de las características demográficas y clínicas de la población. Se utilizó la prueba de ji al cuadrado para variables categóricas y la prueba $U$ de Mann-Whitney para variables cuantitativas. Se realizó una regresión logística para evaluar las diferencias en los desenlaces primarios entre los periodos analizados, ajustados para edad, sexo y las variables con $p<0.1$ en el análisis univariado. Se considero un valor de $p<0.05$ como estadísticamente significativo. El análisis fue realizado en SPSS v20 (IBM Corporation, Armonk, NY).

\section{Resultados}

Se incluyeron 598 pacientes, 352 de la fase 2 y 246 de la fase 1. De ellos, 346 (57.8 \%) eran hombres. No 
Tabla 1. Características demográficas y clínicas

\begin{tabular}{|c|c|c|c|}
\hline Características & $\begin{array}{c}\text { Periodo } 1 \text { (pre-UCN) n = 246, } \\
n /{ }^{*}(\%)\end{array}$ & $\begin{array}{c}\text { Periodo } 2 \text { (UCN) } n=352 \\
n /{ }^{*}(\%)\end{array}$ & p \\
\hline Edad, promedio $\pm \mathrm{DE}$ & $62.97 \pm 13.9$ & $63.17 \pm 14.2$ & $0.921^{\S}$ \\
\hline Hombres & $144(58.5)$ & $202(57.4)$ & 0.423 \\
\hline Hipertensión & $146 / 243(60.1)$ & $242 / 349(69.3)$ & $0.013^{\pi}$ \\
\hline Diabetes mellitus & $114 / 242(47.1)$ & $155 / 350(44.3)$ & 0.276 \\
\hline Dislipidemia & $69 / 242(28.5)$ & $71 / 331(21.5)$ & $0.033^{n}$ \\
\hline Fumador & $75 / 241(31.1)$ & $108 / 350(30.9)$ & 0.508 \\
\hline Ictus previo & $58 / 243(23.9)$ & $82 / 350(23.4)$ & 0.489 \\
\hline Cardiopatía $^{\dagger}$ & $48 / 243(19.8)$ & $70 / 351(19.9)$ & 0.520 \\
\hline Índice Modificado de Charlson $\geq 2$ & $189 / 243(77.8)$ & $279 / 349(79.9)$ & 0.296 \\
\hline $\begin{array}{l}\text { Tiempo hasta la admisión } \\
<3 \text { horas } \\
\text { 3-6 horas } \\
\text { 6-24 horas } \\
\text { 1-7 días } \\
>7 \text { días }\end{array}$ & $\begin{array}{c}39 / 241(16.2) \\
35 / 241(14.5) \\
101 / 241(41.9) \\
60 / 241(24.9) \\
6 / 241(2.5)\end{array}$ & $\begin{array}{c}76 / 348(21.8) \\
59 / 348(17) \\
123 / 348(35.3) \\
80 / 348(23) \\
10 / 348(2.9)\end{array}$ & $\begin{array}{l}0.088 \\
0.627 \\
0.106 \\
0.592 \\
0.778\end{array}$ \\
\hline $\begin{array}{l}\text { Etiología } \\
\text { Aterosclerosis } \\
\text { Enfermedad de pequeño vaso } \\
\text { Cardioembólico } \\
\text { No determinado } \\
\text { Otras causas }^{\ddagger}\end{array}$ & $\begin{array}{c}94 / 241(39) \\
46 / 241(19.1) \\
27 / 241(11.2) \\
70 / 241(29) \\
4 / 241(1.6)\end{array}$ & $\begin{array}{c}117 / 337(34.7) \\
59 / 337(17.5) \\
64 / 337(19) \\
89 / 337(26.4) \\
8 / 337(2.4)\end{array}$ & $\begin{array}{l}0.291 \\
0.235 \\
0.011 \rrbracket \\
0.484 \\
0.552\end{array}$ \\
\hline NIHSS al ingreso, promedio $\pm \mathrm{DE}$ & $9.76 \pm 9.9$ & $8.41 \pm 6.1$ & $0.079^{\S}$ \\
\hline Profilaxis para TVP & $132 / 240(55)$ & $265 / 326(81.3)$ & $<0.001^{\Uparrow}$ \\
\hline Complicaciones intrahospitalarias & $23 / 240(9.6)$ & $41 / 338(12.1)$ & 0.205 \\
\hline Muertes intrahospitalarias & $7 / 246(2.8)$ & $17 / 352(4.8)$ & 0.158 \\
\hline Estancia hospitalaria $\geq 7$ días & $115 / 232(49.6)$ & 99/335 (29.6) & $<0.001^{\Uparrow}$ \\
\hline Puntaje Rankin $\geq 3$ a 3 meses de seguimiento & $67 / 113(59.3)$ & $81 / 193(42)$ & $0.002^{\Uparrow}$ \\
\hline
\end{tabular}

encontramos diferencias significativas en las características demográficas entre ambos grupos, aunque sí mayor proporción de hipertensos y menos dislipidemia en el periodo con la UCN (Tabla 1).

Durante el periodo 2, con la UCN, hubo mayor uso de profilaxis para la trombosis venosa profunda (TVP) $(p<0.001)$ y mayor definición etiológica de un evento de origen cardioembólico $(p=0.011)$, y se redujo el número de días de la estancia hospitalaria (7.8 vs. $5.9 ; p<0.001$ ). Aunque al egreso hospitalario no encontramos diferencia en la recuperación funcional $(p>0.5)$, al momento de analizar el porcentaje de pacientes con dependencia para sus actividades de la vida diaria ( $m R s \geq 3$ ) a los 3 meses de seguimiento se encontró que fue mayor en el periodo sin la UCN (59.3 vs. $42 \% ; p=0.002$ ) (Figura 1).

El análisis por regresión logística mantuvo la asociación entre el periodo con la UCN y el aumento en el uso de la profilaxis para TVP (razón de momios [RM]: 3.235; intervalo de confianza del $95 \%$ [IC $95 \%$ ]: 2.189-4.801; $p<0.001$ ), una disminución en los días de estancia hospitalaria (OR: 0.42; IC $95 \%$ : 0.28$0.62 ; p<0.001)$ y una menor proporción de discapacidad funcional grave al seguimiento a los 90 días del egreso (RM: 0.49; IC $95 \%$ : 0.28-0.87; $p=0.015$ ) (Tabla 2). 


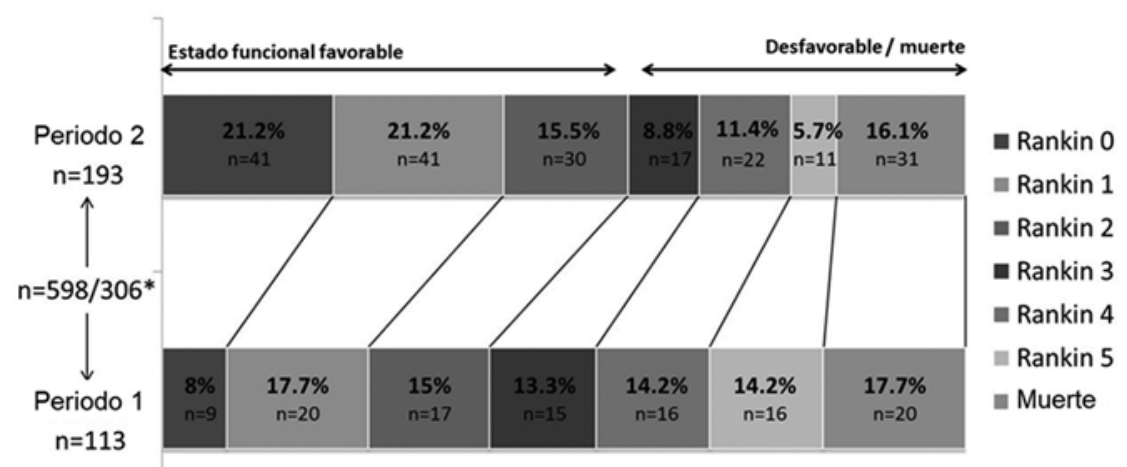

Evaluación de Rankin a los 3 meses.

Figura 1. Número de pacientes con seguimiento a 3 meses. Periodo 1: anterior a la implementación de la unidad de cuidados neurovasculares (UCN). Periodo 2: posterior a la implementación de la UCN.

Tabla 2. Variables asociadas a la implementación de la unidad de cuidados neurovasculares

\begin{tabular}{lcccc}
\hline Variable & p & $\begin{array}{c}\text { RM } \\
\text { ajustada }\end{array}$ & \multicolumn{2}{c}{ IC 95\% } \\
\cline { 4 - 6 } & & & Inferior & Superior \\
\hline Puntaje Rankin $\geq 3$ & $0.015^{*}$ & 0.492 & 0.278 & 0.871 \\
Profilaxis TVP & $<0.001^{*}$ & 3.235 & 2.180 & 4.801 \\
Estancia hospitalaria $\geq 7$ días & $<0.001^{*}$ & 0.419 & 0.285 & 0.617 \\
\hline${ }^{*} p<0.05$. & & & & \\
IC: intervalo de confianza; RM: razón de momios; TVP: trombosis venosa profunda.
\end{tabular}

\section{Discusión}

Nuestro estudio demostró que el ingreso de pacientes a una UCN está asociado a un mejor desenlace funcional y a una estancia hospitalaria más corta. Sin embargo, no hubo diferencias significativas en la frecuencia de administración de terapia con aPTr ni en la mortalidad intrahospitalaria. Diversos metaanálisis han mostrado una mejora en el estado funcional a los 90 días y hasta 1 año posterior al egreso. ${ }^{4}$ En países en vías de desarrollo, un desenlace funcional favorable es especialmente relevante, ya que de lo contrario impacta dramáticamente en la economía familiar. ${ }^{10,11}$

La mejoría en el desenlace funcional puede explicarse porque el ingreso y la estancia en una UCN incluyen la monitorización continua de los signos vitales, resultando en una mayor capacidad para brindar atención oportuna a alteraciones asociadas a desenlaces desfavorables, como arritmias, alteraciones en la presión arterial o incremento en la temperatura. ${ }^{12-14}$ Además, la admisión a una UCN conlleva un contacto más estrecho con el equipo de profesionales de salud, detectando deterioros clínicos más temprano e incrementando medidas de prevención como la evaluación de la disfagia y la profilaxis de la TVP; además, confiere efectos psicológicos benéficos con un efecto favorable en el desenlace..$^{15,16}$

A pesar del incremento en el número de administraciones de aPTr durante el segundo periodo, no hubo diferencia significativa entre ambos periodos. La administración de aPTr debe ser durante las primeras 4.5 horas del inicio de los síntomas del ictus isquémico y no depende de contar con una UCN; en cambio, el incremento en su administración se relaciona con campañas de promoción de la salud y con el uso de la llamada prehospitalaria por las ambulancias. ${ }^{7,17}$

No encontramos una diferencia en la mortalidad intrahospitalaria posterior a la implementación de la UCN. Este hallazgo es similar a estudios previos con una metodología similar a la nuestra. ${ }^{18,19}$ Sin embargo, existen reportes sobre la reducción de la mortalidad en la UCN. ${ }^{20}$ Un metaanálisis reportó que el beneficio en la mortalidad y el grado de dependencia funcional puede verse a 1 año, ${ }^{4}$ sugiriendo que el principal impacto de la UCN es a largo plazo. Diversos factores podrían explicar el efecto neutral de las UCN sobre la mortalidad intrahospitalaria en nuestro estudio. Por ejemplo, las características de la población pueden ser diferentes de las reportadas en la literatura. Comparando con otros estudios, ${ }^{18,21}$ la edad promedio de nuestra población fue menor, pero hubo una mayor prevalencia de comorbilidad como hipertensión y diabetes.

El infarto cerebral de origen cardioembólico representa el de mayor recurrencia y se relaciona con un peor pronóstico funcional a largo plazo. ${ }^{22}$ En nuestro estudio, durante el periodo con la UCN fue posible realizar monitoreo electrocardiográfico continuo y el abordaje necesario para incrementar la detección de alteraciones del ritmo cardiaco, lo cual permitió identificar y prevenir nuevos eventos. 
La disminución en la estancia hospitalaria es concordante con reportes previos. ${ }^{4}$ Esta reducción resultó significativa incluso considerando el mayor índice de comorbilidad en esta población en comparación con la de otros estudios. ${ }^{18,21}$ Por lo tanto, las complicaciones y los costos derivados de una mayor estancia hospitalaria pueden ser reducidos con el uso de UCN, dando como resultado un impacto favorable en la economía del Sistema Nacional de Salud y también en la del propio paciente. ${ }^{11}$ La disminución en los días de estancia hospitalaria es de gran relevancia en los países latinoamericanos y en vías de desarrollo, ${ }^{23}$ donde existe una gran demanda de pacientes que requieren hospitalización y se trabaja con limitaciones presupuestales.

Nuestro estudio tuvo algunas limitaciones. No se puede descartar el impacto de diversos factores de confusión, en especial por el diseño antes y después de esta cohorte prospectiva, y porque el seguimiento de los pacientes se limita a los 3 meses posteriores al egreso hospitalario. Además, debido a que se trata de un solo centro, el grado en el cual estos resultados pueden ser generalizados a otros hospitales de México o de otras regiones con una economía similar es desconocido.

\section{Conclusión}

La implementación de una UCN en un hospital académico de tercer nivel en México mejoró el desenlace funcional y disminuyó los días de estancia intrahospitalaria de los pacientes con ictus isquémico. Estos resultados sugieren que los beneficios de una UCN pueden ser extendidos a otros hospitales del país con recursos limitados, y que la UCN podría ser considerada el estándar de cuidado para el ictus. Además, debido a que las UCN incrementan el volumen de pacientes ingresados, se espera una mejoría en los desenlaces funcionales. ${ }^{24}$ Son necesarias estrategias que faciliten la implementación de las UCN en México y en los países en vías de desarrollo.

\section{Agradecimientos}

Agradecemos a las autoridades de la Facultad de Medicina, del Hospital Universitario Dr. José E. González y del Departamento de Neurología por su ayuda en la conducción de este estudio y facilitar el espacio para la implementación de la UCN.

\section{Bibliografía}

1. Yaggi $H$, Mohsenin V. Obstructive sleep apnoea and stroke. Lancet Neurol. 2004;3:333-42.

2. Márquez-Romero JM, Arauz A, Góngora-Rivera F, Barinagarrementeria F, Cantu C. The burden of stroke in Mexico. Int J Stroke. 2015;10:251-2.

3. Feigin VL, Krishnamurthi R, Bhattacharjee R, Parmar P, Theadom A, Hussein $\mathrm{T}$, et al. New strategy to reduce the global burden of stroke. Stroke. 2015;46:1740-7.

4. Stroke Unit Trialists' Collaboration. Organised inpatient (stroke unit) care for stroke. Cochrane Database Syst Rev. 2013;(9):CD000197.

5. Seenan $\mathrm{P}$, Long $\mathrm{M}$, Langhorne $\mathrm{P}$. Stroke units in their natural habitat: systematic review of observational studies. Stroke. 2007;38:1886-92.

6. Gilligan AK, Thrift AG, Sturm JW, Dewey HM, Macdonell RAL, Donnan GA. Stroke units, tissue plasminogen activator, aspirin and neuroprotection: which stroke intervention could provide the greatest community benefit? Cerebrovasc Dis. 2005;20:239-44

7. Jauch EC, Saver JL, Adams HP, Bruno A, Connors JJB, Demaerschalk BM, et al. Guidelines for the early management of patients with acute ischemic stroke: a guideline for healthcare professionals from the American Heart Association/American Stroke Association. Stroke. 2013;44:870-947.

8. Herrera AL, Góngora-Rivera F, Muruet W, Villarreal HJ, Gutiérrez-Herrera M, Huerta $L$, et al. Implementation of a stroke registry is associated with an improvement in stroke performance measures in a tertiary hospital in Mexico. J Stroke Cerebrovasc Dis. 2015;24:725-30.

9. Wardlaw JM, Sandercock PA, Warlow CP, Lindley RI. Trials of thrombolysis in acute ischemic stroke: does the choice of primary outcome measure really matter? Stroke. 2000;31:1133-5.

10. Mapulanga M, Nzala S, Mweemba C. The socio-economic impact of stroke on households in Livingstone district, Zambia: a cross-sectional study. Ann Med Health Sci Res. 2014;4:123-7.

11. Luengo-Fernández R, Silver LE, Gutnikov SA, Gray AM, Rothwell PM. Hospitalization resource use and costs before and after TIA and stroke: results from a population-based cohort study (OXVASC). Value Heal. 2013;16:280-7.

12. Qin D, Leef G, Alam MB, Rattan R, Munir MB, Patel D, et al. Patient outcomes according to adherence to treatment guidelines for rhythm control of atrial fibrillation. J Am Heart Assoc. 2015;4. pii: e001793.

13. Jorgensen HS, Nakayama H, Olsen TS, Raaschou HO. Effect of blood pressure and diabetes on stroke in progression. Lancet. 1994;344:156-9.

14. Azzimondi G, Bassein L, Nonino F, Fiorani L, Vignatelli L, Re G, et al. Fever in acute stroke worsens prognosis. A prospective study. Stroke. 1995;26:2040-3.

15. Indredavik B, Bakke F, Solberg R, Rokseth R, Haaheim LL, Holme I. Benefit of a stroke unit: a randomized controlled trial. Stroke. 1991;22:1026-31.

16. Indredavik B, Bakke F, Slordahl SA, Rokseth R, Hâheim LL. Treatment in a combined acute and rehabilitation stroke unit: which aspects are most important? Stroke. 1999;30:917-23.

17. Uehara T, Kimura K, Okada Y, Hasegawa Y, Tanahashi N, Suzuki A, et al. Factors associated with onset-to-door time in patients with transient ischemic attack admitted to stroke centers. Stroke. 2014;45:611-3.

18. Gattellari M, Worthington J, Jalaludin B, Mohsin M. Stroke unit care in a real-life setting: can results from randomized controlled trials be translaed into every-day clinical practice? An observational study of hospital data in a large australian population. Stroke. 2009;40:10-7.

19. Nasi LA, Ferreira-Da-Silva AL, Martins SCO, Furtado MV, Almeida AG, Brondani $R$, et al. Implementation of a dedicated cardiovascular and stroke unit in a crowded emergency department of a tertiary public hospital in Brazil: effect on mortality rates. Acad Emerg Med. 2014;21:40-6.

20. Suwanwela NC, Eusattasak N, Phanthumchinda K, Piravej K, Locharoenkul $\mathrm{C}$. Combination of acute stroke unit and short-term stroke ward with early supported discharge decreases mortality and complications after acute ischemic stroke. J Med Assoc Thail. 2007:90:1089-96.

21. Tamm A, Siddiqui M, Shuaib A, Butcher K, Jassal R, Muratoglu M, et al. Impact of stroke care unit on patient outcomes in a community hospital. Stroke. 2014;45:211-6.

22. Arboix A, Alioc J. Cardioembolic stroke: clinical features, specific cardiac disorders and prognosis. Curr Cardiol Rev. 2010;6:150-61.

23. Borghans I, Heijink R, Kool T, Lagoe RJ, Westert GP. Benchmarking and reducing length of stay in Dutch hospitals. BMC Health Serv Res. 2008;8:220.

24. Svendsen ML, Ehlers LH, Ingeman A, Johnsen SP. Higher stroke unit volume associated with improved quality of early stroke care and reduced length of stay. Stroke. 2012;43:3041-5. 